\title{
E-cadherin expression on multiple myeloma cells activates tumor-promoting properties in plasmacytoid DCs
}

\author{
Enguang Bi, ${ }^{1}$ Rong Li, ${ }^{2}$ Laura C. Bover, ${ }^{3}$ Haiyan Li, ${ }^{1}$ Pan Su, ${ }^{1}$ Xingzhe Ma, ${ }^{1}$ Chunjian Huang, ${ }^{1}$ Qiang Wang, ${ }^{1}$ Lintao Liu, ${ }^{1}$ \\ Maojie Yang, ${ }^{1}$ Zhijuan Lin, ${ }^{1}$ Jianfei Qian, ${ }^{1}$ Weijun Fu, ${ }^{2}$ Yong-Jun Liu, ${ }^{4}$ and Qing Yi ${ }^{1}$ \\ 'Department of Cancer Biology, Lerner Research Institute, Cleveland Clinic, Cleveland, Ohio, USA. Department of Hematology, Changzheng Hospital, Second Military Medical University, Shanghai, China. \\ ${ }^{3}$ Department of Cenomic Medicine, Division of Cancer Medicine, The University of Texas MD Anderson Cancer Center, Houston, Texas, USA. ${ }^{4}$ \& D Sanofi, Cambridge, Massachusetts, USA.
}

\begin{abstract}
Plasmacytoid dendritic cells (pDCs) play a key role in antiviral responses by producing type-1 IFNs. However, recent studies showed that pDCs induce immune suppression and promote tumor growth in human ovarian cancer and myeloma. The molecular mechanisms underlying pDC acquisition of these properties are unknown. Here we show that human pDCs activated by $\mathrm{CpC}$ inhibited growth and induced apoptosis in myeloma cells via secreted IFN- $\alpha$, but direct contact with myeloma cells converted pDCs into tumor-promoting cells by suppressing pDC IFN- $\alpha$ production. E-cadherin, expressed on both myeloma cells and pDCs, mediated these effects via a homophilic interaction - activation of E-cadherin signaling upregulated and activated TNFAIP3 to interact with TLR9, resulting in TLR9 ubiquitination and degradation, and inhibition of IFN- $\alpha$ production in pDCs. These findings were supported by an in vivo study in which pDC depletion induced tumor regression and better survival in the Vk*MYC myeloma mouse model. Furthermore, IFNAR1 expression level positively correlated to overall survival of patients with multiple myeloma (MM), and the IFN- $\alpha$ level in patient bone marrow was significantly lower than that in marrow of healthy individuals. This study reveals a novel mechanism underlying how MM tumors educate pDCs in their microenvironment and provides new targets for improving the treatment of MM.
\end{abstract}

\section{Introduction}

Multiple myeloma (MM) is a plasma cell neoplasia accounting for about $10 \%$ to $15 \%$ of all hematologic malignancies (1). Under physiological conditions, the germinal center response results in the differentiation of antibody-secreting plasma cells to mediate a humoral immune response (2). In MM, however, due to abnormal genetic and microenvironment changes, plasma cells can transform into a malignant neoplasm. MM development includes different stages, from a monoclonal gammopathy of undetermined significance (MGUS) to smoldering myeloma and finally to symptomatic myeloma (3). The general mechanisms by which tumors escape from immune surveillance include reduced expression of tumor antigens and HLA molecules, increased expression of inhibitory ligands such as programmed cell death ligand 1 (PDL1), and recruitment of regulatory T cells (Tregs) and myeloidderived suppressor cells (MDSCs) $(4,5)$. However, it is unclear how immune evasion happens during myeloma pathogenesis.

$\mathrm{Vk}^{*} \mathrm{MYC}$ mice were generated by M. Chesi et al. and all mice developed myeloma with age (6). It was reported that $\mathrm{Vk}^{\star} \mathrm{MYC}$ mice share many of the important clinical features of human MM.

Authorship note: EB and RL contributed equally to this work. Conflict of interest: The authors have declared that no conflict of interest exists. License: Copyright 2018, American Society for Clinical Investigation.

Submitted: March 30, 2018; Accepted: August 21, 2018.

Reference information: / Clin Invest. 2018;128(11):4821-4831.

https://doi.org/10.1172/JCI121421.
First, the monoclonal paraproteins in $\mathrm{Vk}^{*} \mathrm{MYC}$ mice are all isotype class-switched, and secreted at a level similar to human disease. Second, $\mathrm{Vk}^{*} \mathrm{MYC}$ mice have an indolent course which results in end organ damage such as renal dysfunction, bone disease, and anemia. Syngeneic recipient mice will develop MM after $\mathrm{Vk}^{*} \mathrm{MYC}$ $\mathrm{MM}$ cell transplantation and the isotype of monoclonal gammopathy is mainly IgG1 and IgG3 (6). These features make it a good model to study human MM. The development of new drugs such as bortezomib and lenalidomide has continuously improved the survival of patients with MM. However, MM still remains incurable because tumors eventually develop resistance to all current treatments (7). In addition to intrinsic MM drug resistance, interaction between $\mathrm{MM}$ cells and bone marrow microenvironment cells, such as plasmacytoid dendritic cells (pDCs) (8), macrophages (9), MDSCs $(10,11)$, osteoclasts (OCs) (12), and bone marrow stromal cells (BMSCs) (13), promotes MM cell growth and survival by inducing drug resistance in patients with MM.

pDCs are a distinct DC subset that have plasmacytoid morphology and abundant endoplasmic reticulum (14). Human pDCs selectively express intracellular TLR7 and TLR9, which can recognize virus-associated nucleic acids such as single-stranded RNA and unmethylated CpG-containing DNA (CpG), respectively (1517). Although most cells can secrete IFN- $\alpha$ in vivo, pDCs produce large amounts of IFN- $\alpha$ compared with other cells after stimulation. MHC expression is low on pDCs, but pDCs still possess antigen presentation ability (15). These characteristics enable pDCs to play a central role in antivirus innate immunity and bridge innate 
A

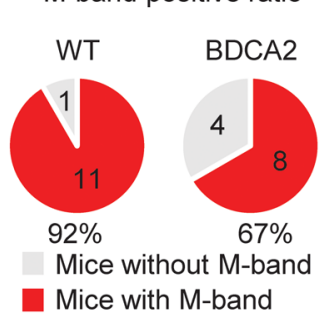

B

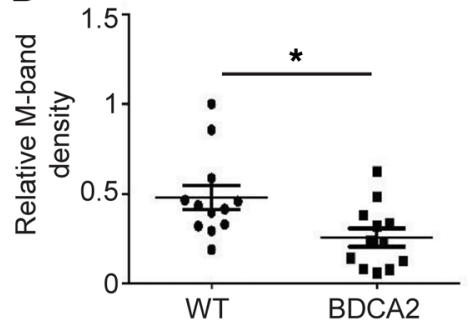

C

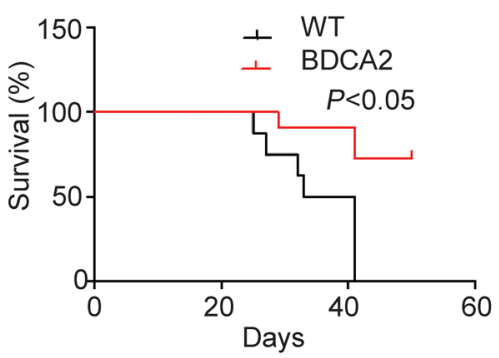

D

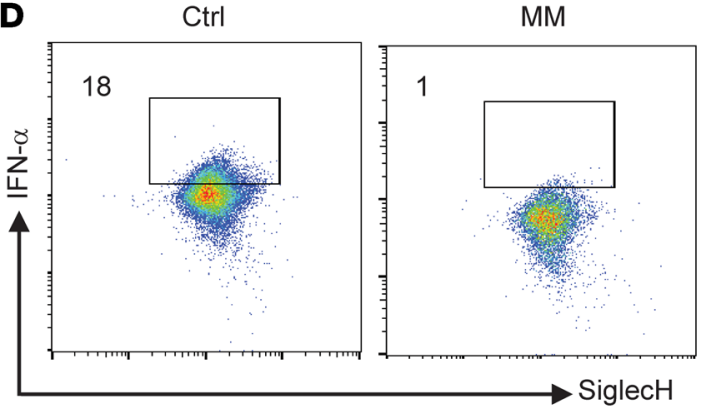

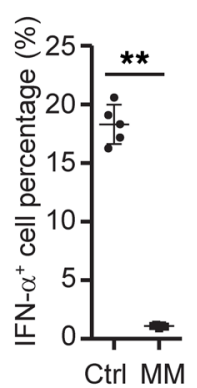

Ctrl MM

E
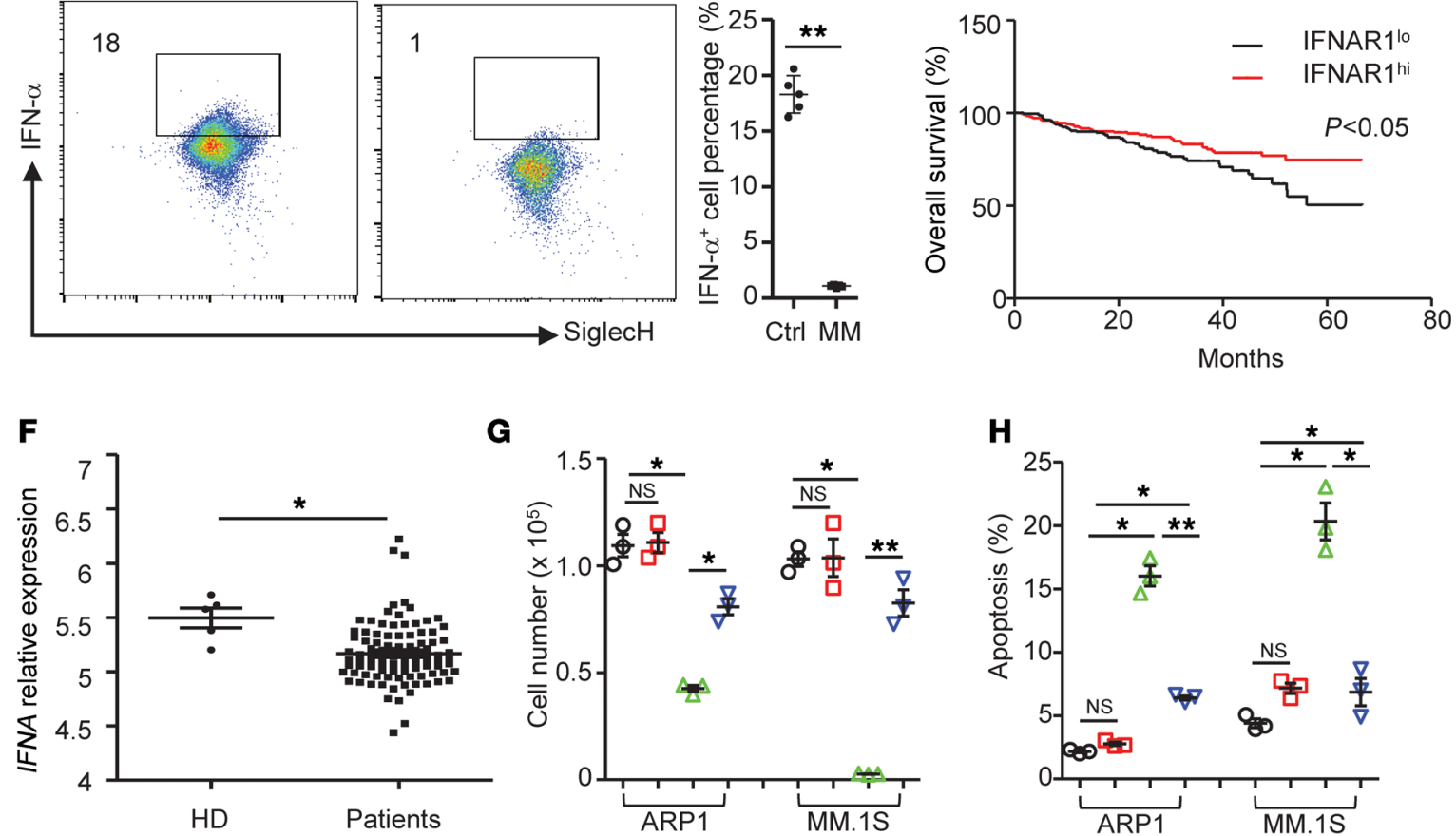

G
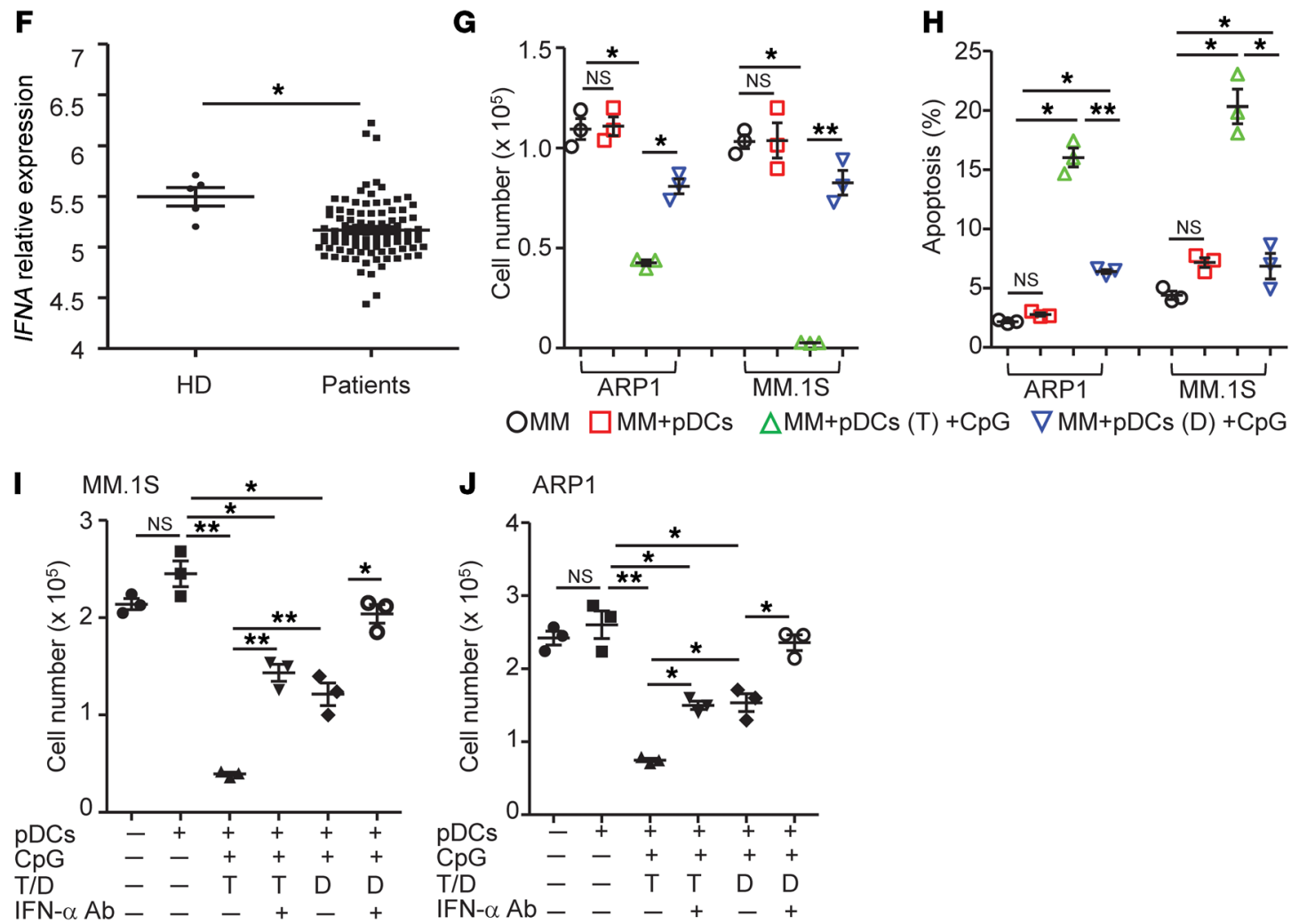

Figure 1. MM cell-conditioned pDCs promote tumor growth in vivo. BDCA2-DTR mice $(n=12)$ and WT littermates $(n=12)$ were injected i.p. with DT (100 ng/mouse) 1 day before i.v. injection of Vk* MYC myeloma cells. DT was administrated every other day for 5 times. Blood was collected weekly via tail vein for detection of the monoclonal band (M-band) using serum protein electrophoresis. Shown are (A) the positive ratio of mice with M-band, (B) quantified relative M-band density, and (C) mouse survival. (D) Splenocytes from tumor-free (Ctrl) or myeloma-bearing (MM) WT mice were stimulated with CpC and blocked with Brefeldin A. IFN- $\alpha$ production was detected in pDC cells by FACS and quantified. (E) Overall survival of patients with MM based on high IFNAR1 (IFNAR1 ${ }^{\text {il) }}$ and low IFNAR1 (IFNAR1 ${ }^{\text {lo) }}$ ) gene expression (CSE2658 data set). (F) Levels of IFN- $\alpha$ expression in bone marrow from healthy donors $(n=5 ;$ HD) and patients with MM ( $n=100)$. MM (ARP1 and MM.1S) cells were cultured alone or in direct (D) or transwell (T) coculture with pDCs (freshly sorted human pDCs from blood of healthy donors; the same thereafter unless otherwise stated) with or without CpG. (C) The number of live MM cells and (H) MM cell apoptosis are shown. Number of live MM.1S cells (I) and ARP1 cells (J) cultured alone, or in direct (D) or transwell (T) coculture with $\mathrm{pDC}$ s with or without $\mathrm{CpC}$, in the presence or absence of IFN- $\alpha$-neutralizing mAb. Experiments were performed 3 times in A-D and G-I. Statistical significance was obtained by Student's $t$ test, and Bonferroni's corrected significance level was used when more than 2 groups were included in an analysis. ${ }^{*} P<0.05,{ }^{* *} P<0.01$. 
and adoptive immunity. pDCs have been reported to accumulate in the bone marrow of patients with MM, and these pDCs were unable to stimulate $\mathrm{T}$ cell proliferation but promoted myeloma cell growth and survival (8). Therefore, we sought to understand how MM microenvironment or MM cells condition normal pDCs to become tumor-promoting cells. We show that MM cells can directly convert $\mathrm{pDCs}$ into a protumor phenotype with decreased production of IFN- $\alpha$ and reduced expression of cell surface costimulatory molecules. Direct coculture with MM cells via surface E-cadherin (CDH1) dramatically reduced pDC ability to secrete IFN- $\alpha$ because activation of CDH1 signaling downregulated TLR9 expression by TNFAIP3 (A20) and greatly attenuated TLR9 signaling. Our study elucidated what we believe is a novel mechanism underlying MM cell escape from immune surveillance mediated by pDCs, and provides a new target to improve the treatment of human MM.

\section{Results}

$M M$ is associated with deficient IFN- $\alpha$ signaling in pDCs. To investigate the role of $\mathrm{pDCs}$ in MM pathogenesis, we injected $\mathrm{Vk}^{*} \mathrm{MYC}$ MM cells (18) into WT or BDCA2-DTR C57BL/6 mice (Supplemental Figure 1A; supplemental material available online with this article; https://doi.org/10.1172/JCI121421DS1), which express the diphtheria toxin receptor and can be depleted of pDCs by administration of diphtheria toxin (DT). At 4 weeks after tumor injection, myeloma became established, evident by the appearance of a dominant monoclonal Ig band (M-spike protein secreted by myeloma cells; Supplemental Figure 1B). About $92 \%$ of WT mice developed myeloma, whereas only $67 \%$ of pDC-depleted mice developed myeloma (Figure 1A). Furthermore, myeloma-bearing WT mice had a higher M-protein concentration (Figure 1B) and shorter survival (Figure 1C) than pDC-depleted mice. Consistent results were obtained when BDCA2-DTR mice without DT treatment were used as a control (Supplemental Figure 1, C-F). These findings indicated that pDCs play an important role in the pathogenesis of MM in vivo.

Next we examined the phenotype of pDCs in normal and $\mathrm{Vk}^{*} \mathrm{MYC}$ myeloma-bearing mice. Normal pDCs produced large amounts of IFN- $\alpha$ upon $\mathrm{CpG}$ stimulation, whereas cells from myeloma-bearing B6 mice lost the ability to secrete IFN- $\alpha$ (Figure 1D). To determine the clinical relevance of this finding, we analyzed a published patient MM data set from Oncomine. We found that the level of IFNAR1 (interferon alpha and beta receptor subunit 1) expression positively correlated to the overall survival of patients with MM (Figure 1E), and the IFN- $\alpha$ expression in myeloma bone marrow was significantly lower than that in healthy individuals (Figure 1F). These findings suggested that $\mathrm{pDC}$-secreted IFN- $\alpha$ may play an important role in inhibiting MM growth and survival in vivo.

To determine the effect of pDC-derived IFN- $\alpha$ on MM cells, we cocultured human pDCs (freshly sorted from human blood; the same hereafter when pDCs are mentioned) and human MM cells with or without $\mathrm{CpG}$ and examined MM cell growth and apoptosis. In the absence of $\mathrm{CpG}, \mathrm{MM}$ cells grew well (Figure 1G) and did not undergo apoptosis (Figure $1 \mathrm{H}$ ) in culture alone or in direct coculture with pDCs. In the presence of $\mathrm{CpG}, \mathrm{MM}$ cells grew poorly and underwent apoptosis, especially in transwell coculture with pDCs, suggesting that soluble factors secreted by
CpG-activated pDCs inhibit MM growth and induce MM apoptosis, and that secretion of the factors by pDCs was largely inhibited by direct coculture with MM cells. Because CpG activates pDCs to secrete large amounts of IFN- $\alpha$ (19), we examined whether MM growth inhibition and apoptosis were IFN- $\alpha$ dependent. Figure 1, I and J, clearly shows that neutralizing IFN- $\alpha$ effectively restored the number of MM cells in either transwell or direct coculture with pDCs. Taken together, these results show that $\mathrm{CpG}$-activated pDCs are able to induce apoptosis in MM cells indirectly by secreting IFN- $\alpha$, but direct contact with MM cells greatly reduces $\mathrm{pDC}$ ability to produce IFN- $\alpha$.

$M M$ cells, upon direct contact, cause dysfunction in pDCs. Because CpG-activated pDCs effectively killed MM cells in transwell coculture but less so in direct coculture, we hypothesized that $\mathrm{MM}$ cells, upon direct contact, modulate pDC function. To test this hypothesis, $\mathrm{pDCs}$ and MM cells were cocultured under various conditions, and pDC-derived cytokines such as IFN- $\alpha$, TNF- $\alpha$, and IL- 6 were measured. Indeed, MM cells, in direct but not transwell coculture, significantly reduced pDC secretion of IFN- $\alpha$, TNF- $\alpha$, and IL-6 (Figure 2A). To gain a global view of the changes in pDCs induced by MM cells, we performed gene microarray of pDCs under various conditions as described in Methods, and analyzed the data with Ingenuity Pathway Analysis (IPA). A decrease in type-1 IFN pathway transcripts was the top change in pDCs directly cocultured with MM cells (Figure 2B). In line with this result, the expression of type-1 IFN genes and IFN-induced genes decreased strikingly in pDCs in direct coculture with MM cells (Figure 2C). Because pDCs also function as antigen-presenting cells, we analyzed the expression of surface molecules and found that $\mathrm{MM}$ cells also markedly downregulated pDC expression of MHC (HLA-ABC and HLA-DR), CD86, CD40, and PD-L1 (Figure 2D). To determine the clinical significance of this finding, we studied freshly isolated primary $\mathrm{CD} 138^{+} \mathrm{MM}$ cells from patients. Similar to cell lines, primary MM cells also significantly inhibited CpG-induced IFN- $\alpha$ secretion from pDCs, whereas peripheral blood mononuclear cells from healthy donors did not (Figure 2E). We repeated the experiments with a commonly used human pDC like cell line, Gen2.2 (20), and showed that MM cells also inhibited secretion of IFN- $\alpha$ by Gen2.2 cells (Figure 2F).

MM cells suppress type-1 IFN production by downregulating TLR 9 expression in pDCs. Next we elucidated the mechanism underlying MM cell-mediated suppression of IFN- $\alpha$ expression in pDCs. As IFN- $\beta$ is the type- 1 IFN first expressed in response to viral infection and positively regulates IFN- $\alpha$ expression (21), we first examined IFN- $\beta$ production by $\mathrm{pDCs}$ at different time points in culture with or without MM cells. IFN- $\alpha$ production followed similar patterns and kinetics (Figure 3, A and B), suggesting that IFN- $\beta$ was not responsible for regulating IFN- $\alpha$ expression in pDCs in the coculture. Second, because TLR7 and TLR9 are selectively expressed in pDCs to sense viral stimulation to produce IFN- $\alpha$, the role of the TLR7 and TLR9 pathways was determined. Flow analysis showed that TLR9 and TLR7 expression in pDCs was significantly downregulated after direct, but not transwell, coculture with MM cells (Figure 3, C and D). Similarly, MM cells also significantly downregulated TLR9 expression in Gen2.2 cells, and the effect could be observed after only 30 minutes in coculture with MM cells (Supplemental Figure 2A). 

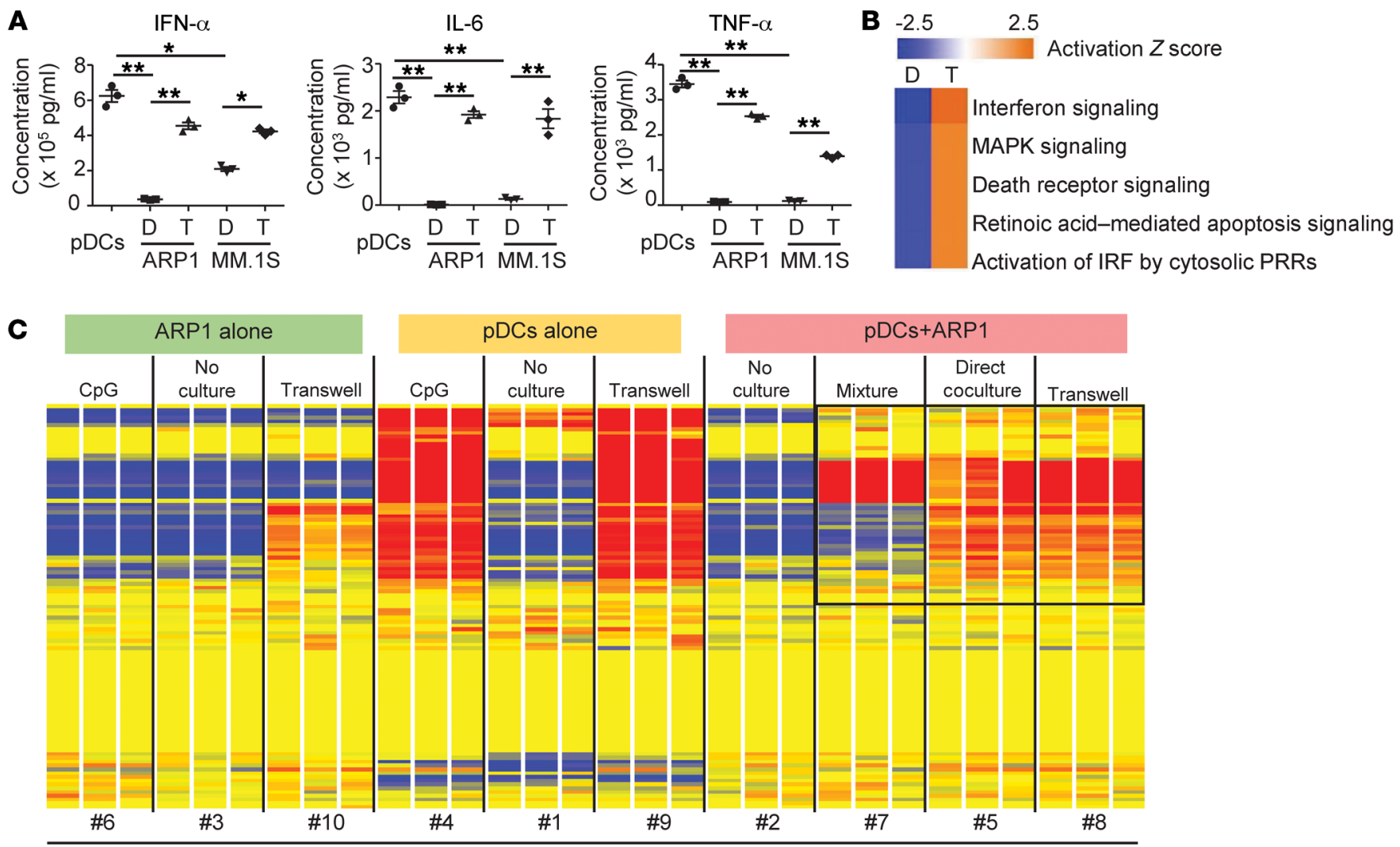

Culture conditions

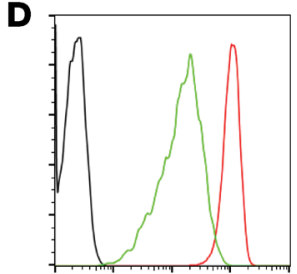

HLA-ABC

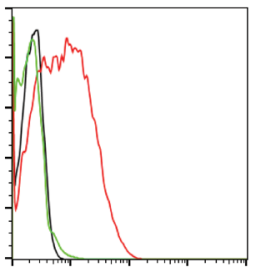

CD40

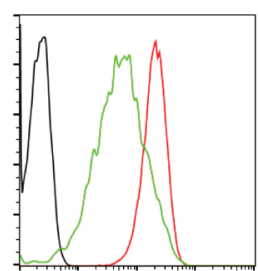

HLA-DR

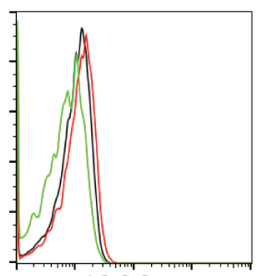

icos

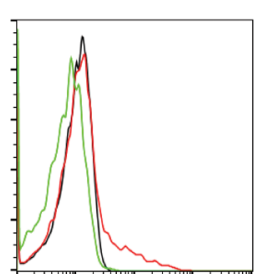

CD80

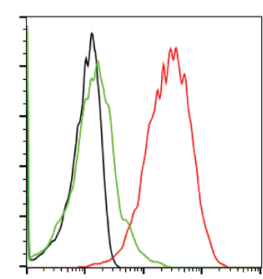

PD-L1

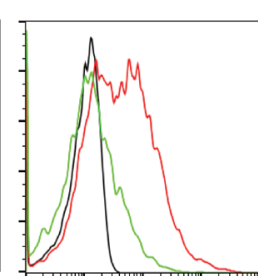

CD86

- Ctrl

_ pDCs

$-\mathrm{pDCs}+\mathrm{MM} .1 \mathrm{~S}$

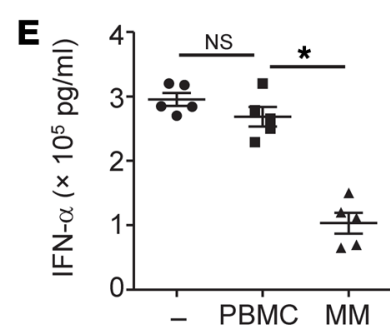

$\mathbf{F}$

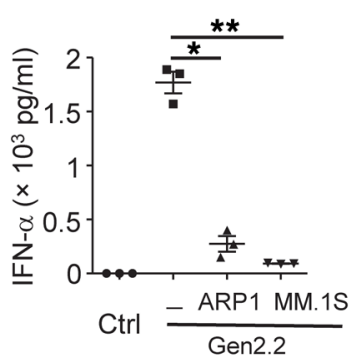

Figure 2. MM cells, via direct contact, inhibit the production of IFN- $\alpha$ and other cytokines by pDCs. (A) Cytokine levels in the supernatants of pDCs cultured alone or in direct (D) or transwell (T) coculture with MM (ARP1 or MM.1S) cells with CpG for 16 hours (the same thereafter, unless otherwise stated). Global gene profiling of pDC culture alone or in coculture with MM (ARP1) cells, and data were analyzed by (B) IPA assay for the canonical pathways, or presented as (C) heatmaps to show the change in activation of IFN genes and IFN-inducible genes under various conditions. Gene profiling of ARP1 cells served as a control. (D) Expression of cell surface molecules on pDCs with or without direct coculture with MM (MM.1S) cells. (E) IFN- $\alpha$ secreted by pDCs (plus $\mathrm{CpG}$ ) in direct coculture with primary MM cells freshly isolated from bone marrow of patients with MM or with PBMCs from healthy donors as a control. (F) Level of IFN- $\alpha$ secreted by the human pDC cell line Gen2.2, cultured alone or in a direct coculture with MM (ARP1 or MM.1S) cells, in the absence (control, Ctrl) or presence of CpG (Gen2.2). Experiments were performed 3 times in $\mathbf{A}$ and $\mathbf{D}-\mathbf{F}$. Statistical significance was obtained by Student's $t$ test, and Bonferroni's corrected significance level was used when more than 2 groups were included in an analysis. ${ }^{*} P<0.05,{ }^{* *} P<0.01$.

To confirm the results, Gen2.2 and ARP1 or MM.1S MM cells were cocultured for 16 hours, followed by depletion of MM cells using CD138 antibody-coated magnetic beads. Consistent with the flow analysis, the expression of TLR7 and
TLR9 in Gen2.2 was significantly and strikingly reduced after coculture with MM cells, as detected by Western blot (Figure $3 E$ ) and quantitative PCR (qPCR) (Figure 3F). The expression of MyD88, the TLR adaptor protein, was also decreased. In line 
A

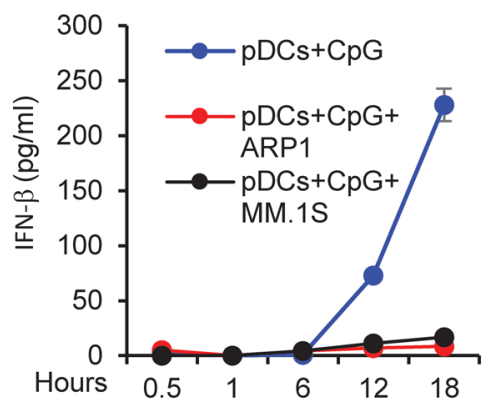

B

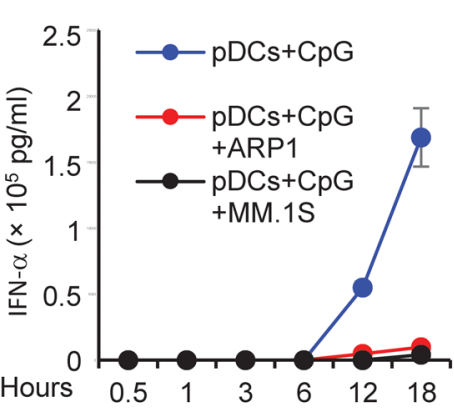

C

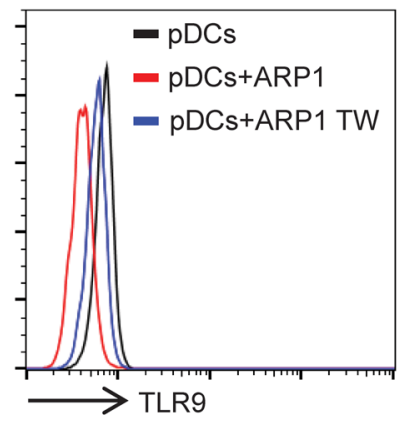

D

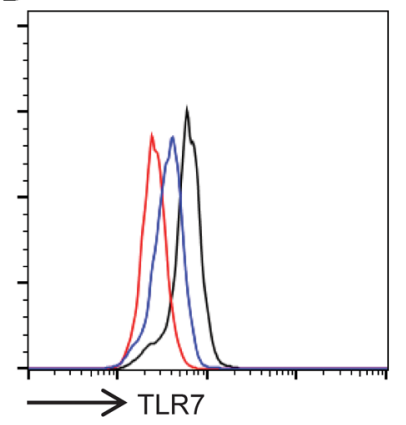

E

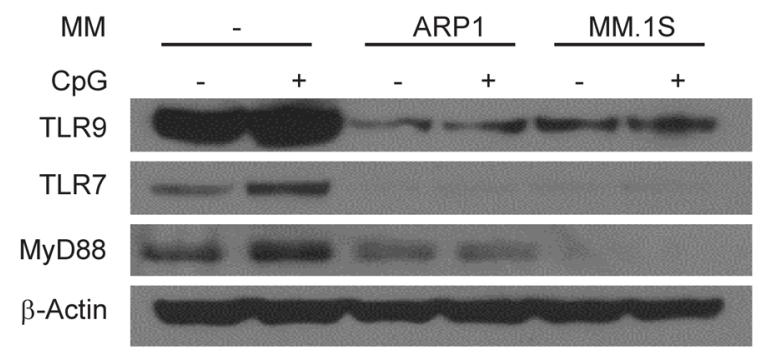

G

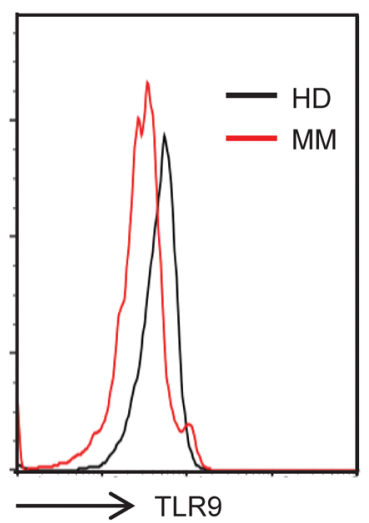

F $\quad T L R 9$
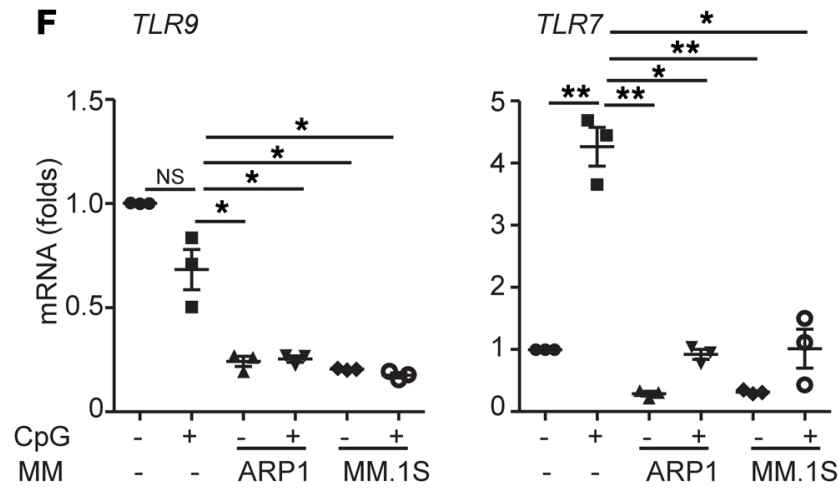

H

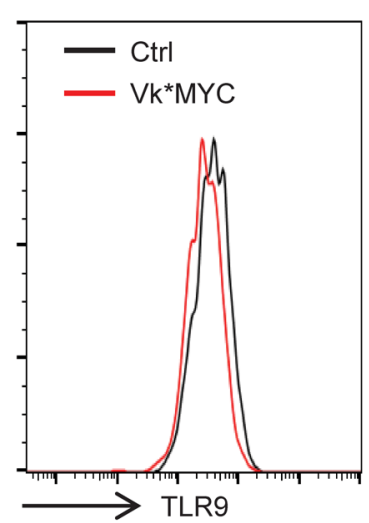

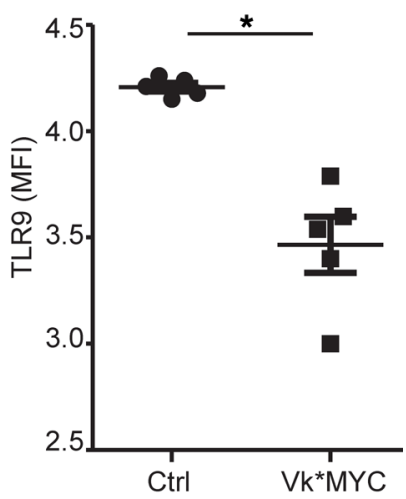

Figure 3. MM cells inhibit pDC IFN- $\alpha$ production by downregulating pDC TLR9 expression. pDCs were cultured alone or in direct coculture with MM (MM.1S or ARP1) cells in the presence of $\mathrm{CpG}$, and supernatant was collected at the indicated time points for measurement of (A) IFN- $\beta$ and (B) IFN- $\alpha$. Expression of (C) TLR9 or (D) TLR7 by (CD123+CD138-) pDCs cultured alone or in direct or transwell (TW) coculture with MM (ARP1) cells in the presence of CpG for 16 hours. Gen2.2 cells were directly cocultured with MM (ARP1 or MM.1) cells with or without CpG for 16 hours, followed by depletion of MM cells with CD138 ${ }^{+}$beads. Gen2.2 cells were collected and subjected to (E) Western blot or (F) qPCR analyses for the expression of TLR9/7 and MyD88. Flow cytometry analysis showing the expression levels of TLR9 in (G) human pDCs from healthy donors $(n=5)$ or patients with MM $(n=5)$, or $(\mathbf{H})$ murine pDCs from tumor-free (control, Ctrl) or Vk*MYC myeloma-bearing B6 mice ( $n=5$, each group). All experiments were performed 3 times. Statistical significance was obtained by Student's $t$ test, and Bonferroni's corrected significance level was used when more than 2 groups were included in an analysis. ${ }^{*} P<0.05,{ }^{* *} P<0.01$.

with these results, microarray data showed that the TLR7/9 signaling pathway was downregulated (Supplemental Figure 2B). We also examined pDCs from patients with MM and healthy donors and found that TLR9 expression was significantly lower in patient pDCs than in those from healthy donors (Figure 3G). Interestingly, pDCs from $\mathrm{Vk}^{*} \mathrm{MYC}$-bearing mice also expressed a lower level of TLR9 than those from tumorfree mice (Figure $3 \mathrm{H}$ ). Taken together, these results clearly show that MM cells inhibited pDC IFN- $\alpha$ secretion by downregulating their TLR9 expression.
$B S T 2$ and ILT7 are involved in MM-pDC interaction but not $p D C$ dysfunction. Because MM cell-induced pDC dysfunction was contact-dependent, we searched for surface molecules on MM cells and pDCs that were interactive and mediated the effects. We focused our attention on the pair of surface molecules BST2 and ILT7 because our previous studies identified ILT7 as a pDC surface receptor that binds with BST2 on many cells, including breast cancer cells, and regulates TLR7/9 responses (22). Based on published data from Oncomine, BST2 is highly expressed on MM and other tumor cells (Supplemental Figure 3, A and B), and ILT7 
is exclusively expressed on pDCs (Supplemental Figure 3C). To determine whether MM cells use this pair of molecules to inhibit pDC function, we generated BST2-knockdown MM cell lines and FceRI $\gamma$-knockdown Gen2.2 cells (FceRI $\gamma$ is the adaptor molecule of ILT7) (Supplemental Figure 3, B and D). To our surprise, knocking down either or both of the molecules on MM cells or pDCs did not abrogate the MM cell-mediated inhibition on pDC secretion of IFN- $\alpha$ (Supplemental Figure 3E), indicating that these surface molecules are not involved in MM cell-induced pDC dysfunction. Nevertheless, BST2 and ILT7 were important for pDC and MM cell interaction and adhesion, because blocking antibodies against either BST2 or ILT7 significantly and substantially decreased the formation and number of pDC and MM cell clusters as detected by flow analysis (Supplemental Figure 3, F and G).

CDH1 mediates MM-induced dysfunction in pDCs. Microarray identified only 4 genes that were changed in all pDC culture conditions with MM cells (direct, mixture, or transwell coculture) (Figure 4A). CDH1, a membrane molecule that mediates cell-cell adhesion by homophilic ligation, was one of the 4 genes identified (23). We wondered whether CDH1 mediates the suppressive effect of MM cells on pDCs. CDH1 is expressed by both pDCs (the Gen2.2 cell line and primary pDCs) and MM cells (cell lines and primary myeloma cells) (Figure 4, B and C). After we knocked down CDH1 in MM cells (Figure 4D), they almost completely lost their ability to inhibit IFN- $\alpha$ secretion (Figure 4E) or downregulate TLR9 expression in pDCs (Figure $4 \mathrm{~F}$ ). However, pDCs were able to inhibit the growth and promote apoptosis of CDH1-knockdown cells but not control MM cells in a direct coculture in the presence of CpG (Supplemental Figure 4). To confirm the results, pDCs were treated with a recombinant CDH1-Fc fusion protein followed by Fc cross-linking antibody. The treatments also inhibited pDC secretion of IFN- $\alpha$ (Figure $4 G$ ). Thus, these results demonstrated that MM cells use their surface CDH1 to inhibit pDC function. As CDH1 often mediates adhesion via homophilic ligation with $\mathrm{CDH} 1$ on other cells, we treated pDCs with CDH1 agonist antibody and observed that the antibody significantly inhibited pDC secretion of IFN- $\alpha$ (Figure 4, H and I). Consistently, CDH1 agonist antibody downregulated the expression of TLR9, MyD88, and IRF7 in treated pDCs (Figure 4J). Taken together, these results indicated that MM cells exert their inhibitory effects on pDCs via CDH1 homophilic interaction.

A2O degrades TLR9 protein in PDCs induced by CDH1 activation and signaling. The observed quick (within 30 minutes) decrease in the protein expression of TLR9 in pDCs after contacting MM cells suggested a degradation of TLR9 protein. We found that lactacystin, a proteasome inhibitor, partially restored pDC IFN- $\alpha$ secretion inhibited by MM cells (Figure 5A), as did CDH1 agonist antibody (Figure 5B), suggesting that TLR9 protein was degraded by the ubiquitin-proteinase pathway. Indeed, activation of CDH1 signaling by the agonist antibody markedly enhanced the ubiquitin of TLR9 in Gen2.2 cells (Figure 5C). A TLR9 pull-down experiment was performed in Gen2.2 cells, followed by mass spectrometry analysis. Among the proteins captured by TLR9 pull down (Figure 5D), TNFAIP3 (A20) is an ubiquitin-editing enzyme with both de-ubiquitination and ubiquitin ligase functions (24), and was increased in pDCs after CDH1 stimulation (Figure 5E). To determine whether A20 was responsible for degrading TLR9, we cotransfected A20 and TLR9 into 293T cells and performed flow cytometry to detect TLR9 expression. As shown in Figure 5F, TLR9 expression gradually decreased as A20 protein expression increased. To determine whether TLR9 and A20 directly interact, TLR9, A20, and $\beta$-catenin (a downstream molecule of CDH1) were cotransfected into $293 \mathrm{~T}$ cells, followed by immunoprecipitation of A20 protein. TLR9 interacted with A20 in the presence, but not absence, of $\beta$-catenin (Figure $5 \mathrm{G}$ ), suggesting that activation of CDH1 signaling is necessary to activate A20 to interact with TLR9. In line with this result, knocking down A20 in primary pDCs greatly and significantly enhanced $\mathrm{CpG}$-induced IFN- $\alpha$ production, confirming that A20 plays an important role in negatively regulating TLR9 expression (Figure 5, H and I). Furthermore, MM cells failed to inhibit $\mathrm{CpG}$-induced IFN- $\alpha$ production by pDCs (Figure 5I) or induce TLR9 degradation (Figure 5J) in pDCs when pDCs were deficient in A20.

\section{Discussion}

We and others have shown that cells in the myeloma bone marrow environment, such as macrophages (9), OCs (12), BMSCs (13), and pDCs (8), promote MM growth and drug resistance. It is unclear how these cells, especially pDCs, acquire a tumor-promoting phenotype and function. Most cells produce type-1 IFNs in response to stimuli (25). Among them, pDCs are the major IFN- $\alpha$-producing cells triggered by TLR7/9 signaling. IFN- $\alpha$, in addition to its direct killing effect on tumor cells, induces tumor immunity by activating immune cells, including cytotoxic $\mathrm{T}$ lymphocytes, natural killer cells, and innate lymphoid cells (26-28), and by negatively regulating immune suppressive cells such as MDSCs (29) and Tregs (30). Our results showed that CpG-activated pDCs directly killed MM cells by secreting IFN- $\alpha$ in vitro, which may suggest the physiological function of pDCs in tumor immune surveillance. However, MM-conditioned pDCs not only lost their ability to secrete IFN- $\alpha$ but also became MM-promoting cells. Indeed, myeloma grew faster in WT $\mathrm{Vk}^{*} \mathrm{MYC}$ mice and decreased mouse survival, whereas in vivo depletion of pDCs in the tumor-bearing mice led to partial tumor regression and improved survival. These results provide what we believe is the first direct evidence to support the role of pDCs in myeloma pathogenesis, and are in line with a previous report showing that pDCs promoted myeloma cell proliferation (8). These findings suggest that MM cells can polarize pDCs into a tumor-promoting phenotype. This may be one of the mechanisms that explains how MM cells escape from immune surveillance mediated by pDCs in vivo. Furthermore, we found that IFNAR 1 expression was positively correlated with the overall survival of patients with MM, and IFN- $\alpha$ was significantly lower in patient bone marrow than in that of healthy donors, indicating that our observations are highly clinically relevant.

IFN- $\alpha$ was used to effectively treat MM and other tumors decades ago; however, the toxicity and a narrow therapeutic index has limited its application. Therefore, restoring the physiological production of IFN- $\alpha$ in patients with MM may be important, and understanding how IFN- $\alpha$ secretion is suppressed in vivo is the first step. Restoring endogenous, physiological production of IFN- $\alpha$ by pDCs may benefit patients with MM in 2 ways. First, high amounts of IFN- $\alpha$ will kill MM cells directly, and second, functional pDCs will not promote MM cell growth. Previous studies reported that 
A

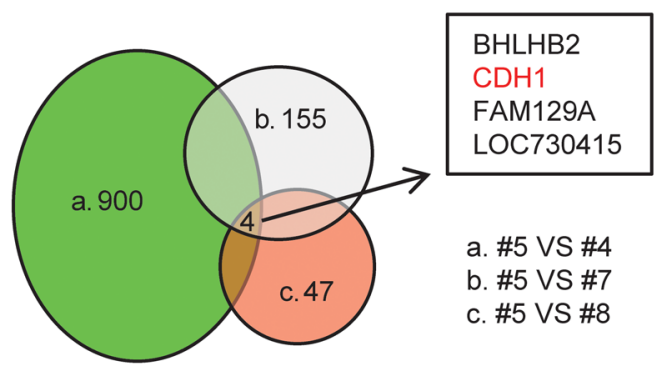

B

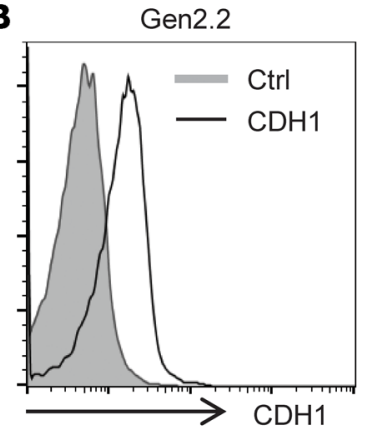

RPMI8226
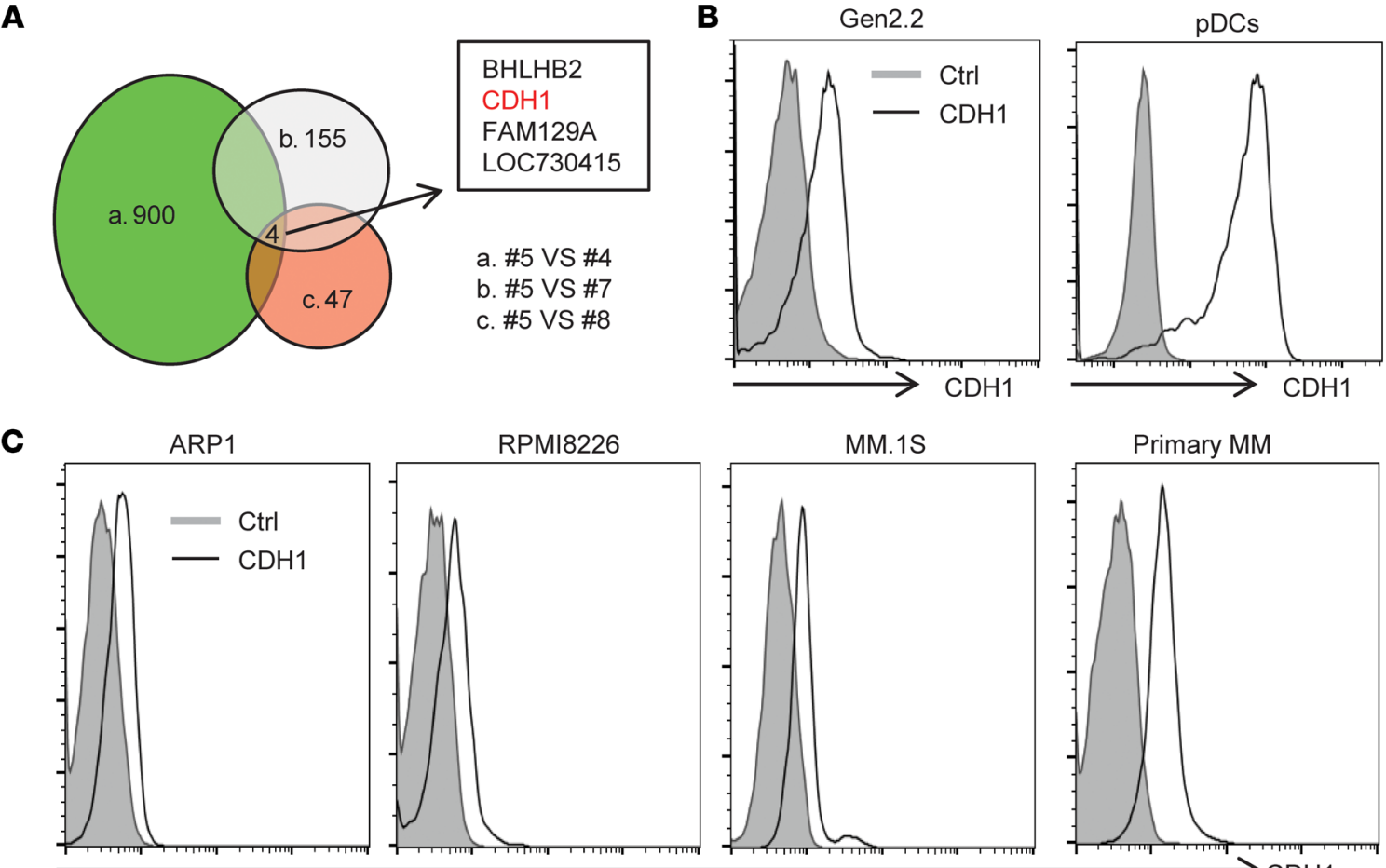

D

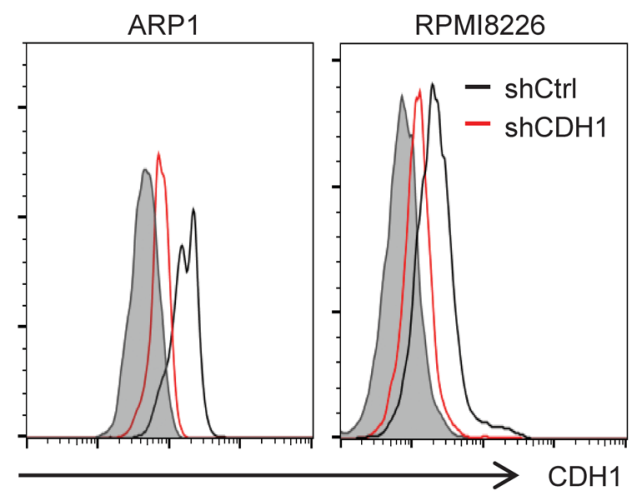

E

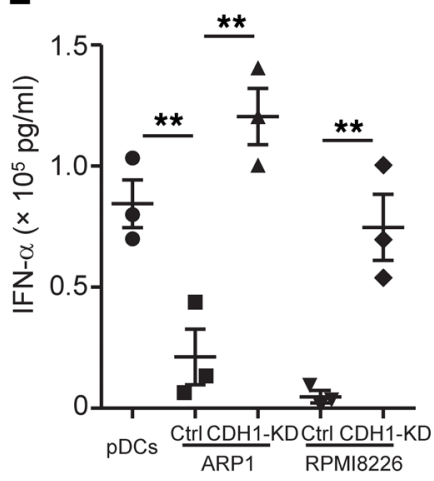

$\mathbf{F}$
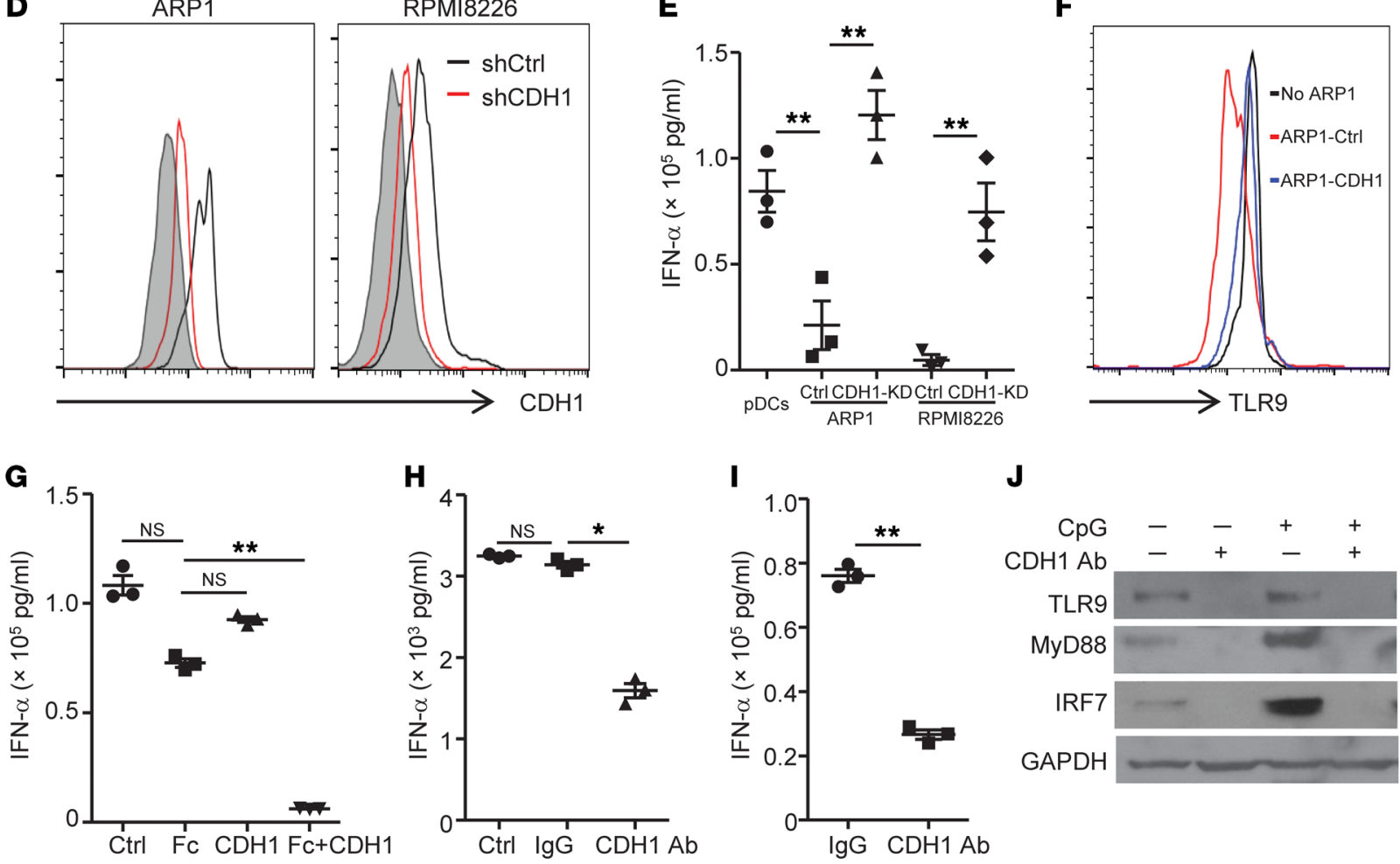

$\mathbf{J}$

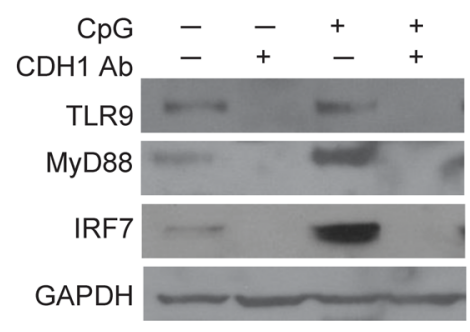

Figure 4. E-cadherin mediates MM-induced dysfunction in pDCs. (A) Venn diagram showing the changes in gene expression in pDCs under various different culture and coculture conditions. Microarray was used to determine the global gene profiling of pDCs. E-cadherin (CDH1) expression on (B) Gen2.2 cells and pDCs, on (C) MM cell lines and primary MM cells, and on (D) ARP1 or RPMI-8226 cells infected with control shRNA (shCtrl) or CDH1 shRNA (shCDH1). (E) Concentration of IFN- $\alpha$ secreted by pDCs (plus CpC) cocultured with control (Ctrl) or CDH1-knockdown (CDH1-KD) MM cells. (F) Expression of TLR9 on pDCs cocultured with control (Ctrl) or CDH1-knockdown MM cells. (C) Concentration of IFN- $\alpha$ secreted by pDCs (plus $\mathrm{CpC}$ ) induced by Fc control or recombinant $\mathrm{Fc}-\mathrm{CDH} 1$ fusion proteins. $\mathrm{CDH} 1$ agonist antibody was used as a positive control. Concentration of IFN- $\alpha$ secreted by (H) Gen2.2 cells or (I) pDCs (plus $\mathrm{CpG}$ ) induced by IgG control or CDH1 agonist antibody. (J) Western blot showing the expression of TLR9, MyD88, and IRF7 by Gen2.2 cells induced by CpC or CDH1 agonist antibody. All experiments except A were performed 4 times. Statistical significance was obtained by Student's $t$ test, and Bonferroni's corrected significance level was used when more than 2 groups were included in an analysis. ${ }^{*} P<0.05,{ }^{* *} P<0.01$. 
A

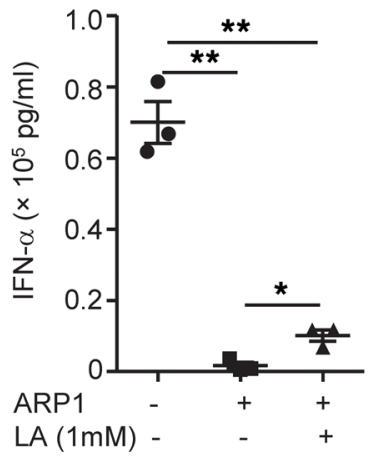

B

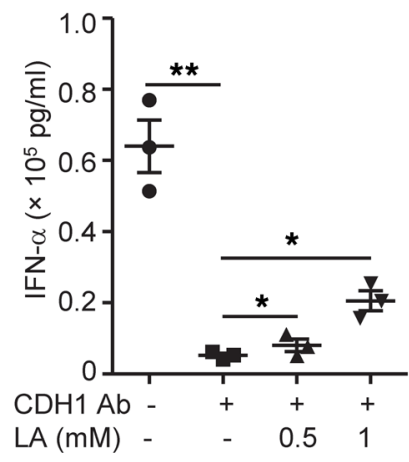

C

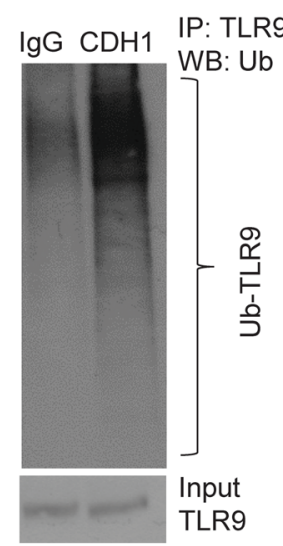

D

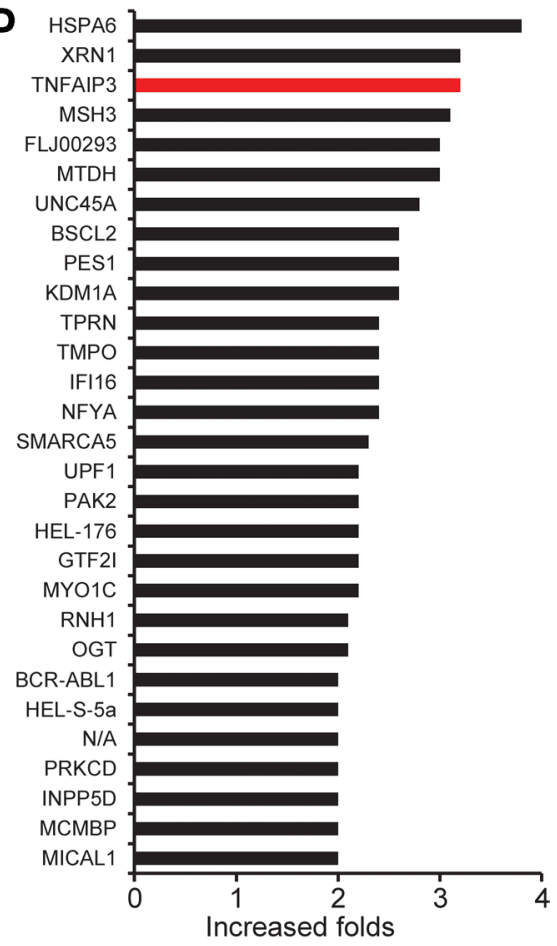

E

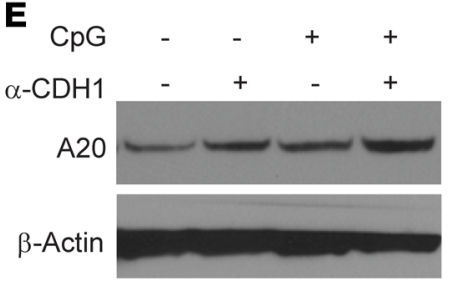

$\mathbf{F}$

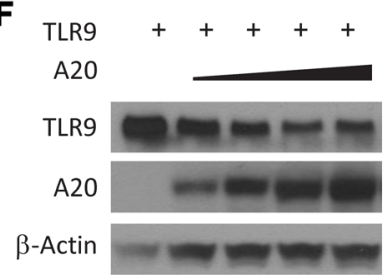

J

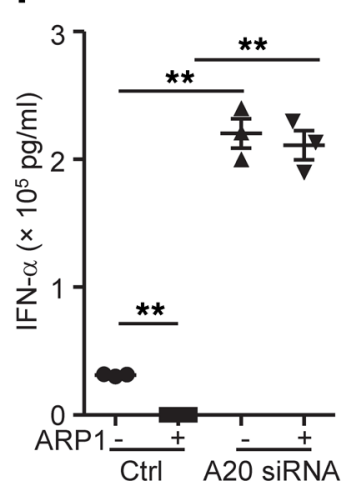

H

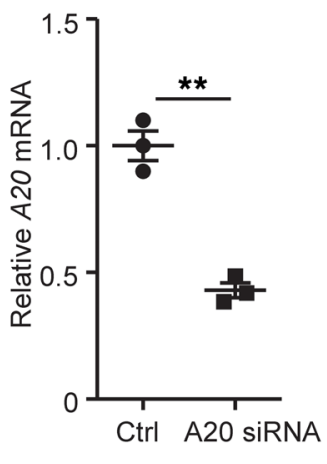

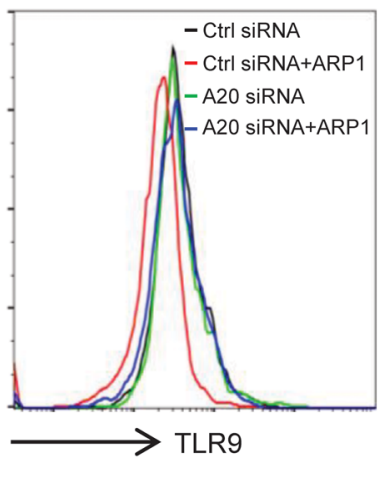

Figure 5. A20 degrades TLR9 protein in pDCs induced by CDH1 activation. Concentration of IFN- $\alpha$ secreted by pDCs (A) in direct coculture with MM (ARP1) cells or (B) induced by CDH1 agonist antibody in the presence of different doses of lactacystin (LA). (C) Immunoprecipitation assay and (D) mass spectrometry showing TLR9 ubiquitination in Gen2.2 (plus CpG) in 1-hour culture with Ig control or CDH1 agonist antibody. Proteins with at least 2-fold increase between CDH1 agonist antibody and control IgG group are shown in (D). Western blot showing (E) expression of A20 in Gen2.2 cells induced by CpG and CDH1 agonist antibody, or (F) expression of TLR9 and A20 in 293T cells cotransfected with TLR9 plasmid and A20 plasmid. Cells were collected 24 hours after transfection and subject to analysis. (G) Immunoprecipitation assay showing the interaction of TLR9 and A20 in $293 T$ cells cotransfected with TLR9 plasmid, A20 plasmid, and $\beta$-catenin plasmid. Cells were harvested 24 hours after transfection and subjected to analysis. pDCs were transfected with control siRNA or A20 siRNA for 24 hours to knockdown A20 (H), and (I) the concentration of IFN- $\alpha$ secreted or (J) expression of TLR9 by control (Ctrl siRNA) or A20 knockdown (A20 siRNA) was determined by using pDCs cocultured with or without MM (ARP1) cells in the presence of CpG. All experiments were performed 3 times. Statistical significance was obtained by Student's $t$ test, and Bonferroni's corrected significance level was used when more than 2 groups were included in an analysis. ${ }^{*} P<0.05,{ }^{* *} P<0.01$

pDCs were dysfunctional in other cancer types such as breast cancer (31) and head and neck cancer (32), which is consistent with our finding. However, all previous studies focused on how pDCs promoted tumor cell proliferation, and no studies had addressed the important questions of what is the phenotype of tumor-associated pDCs and how is it acquired?

PDCs selectively express endosomal TLR7 and TLR9 to sense viral RNA and DNA, respectively, and in response, secrete large amounts of IFNs. This process is tightly regulated. Cell surface mole- cules containing or associated with the ITAM motif play an important and mostly negative role in regulating the TLR7/9 pathway. FceRI $\gamma$ is a common coreceptor for cell surface receptors such as ILT7 and BDCA2. Previous studies indicated that these molecules may regulate IFN- $\alpha$ production in pDCs $(22,33,34)$. In our MM cell-pDC coculture, knock down of either FceRI $\gamma$ or ILT7 ligand (BST2) in MM cells did not restore pDC ability to produce IFN- $\alpha$, indicating that MM cells employ a unique mechanism to interact with pDCs and polarize them to a tumor-promoting phenotype. Until now, it has 


\section{Table 1. Primer sequences used for $q P C R$ and shRNA}

\begin{tabular}{|c|c|}
\hline Primers for qPCR & Sequence (from $5^{\prime}$ to $3^{\prime}$ ) or information \\
\hline$T L R 7$ forward & GGAGGTATTCCCACGAACACC \\
\hline$T L R 7$ reverse & TGACCCCAGTGGAATAGGTACAC \\
\hline TLR9 forward & GGACСTCTGGTACTGCTTCСA \\
\hline TLR9 reverse & AAGCTCGTTGTACACCCAGTCT \\
\hline A20 forward & GACAGCACAATGGCTGAACA \\
\hline A20 reverse & TCCAGTGTGTATCGGTCCAT \\
\hline FCER1G forward & AGTGGTTсСтсСтСССTGTT \\
\hline FCER1G reverse & GTCCAGTCCATGGCAGTTTT \\
\hline ACTB forward & CACCATTGGCAATGAGCGGTTC \\
\hline ACTB reverse & AGGTCTTTGCGGATGTCCACGT \\
\hline \multicolumn{2}{|l|}{ Primers for shRNA } \\
\hline $\mathrm{CDH} 1$ & CCGG AAGATAGGAGTTCTCTGATGC CTCGAG GCATCAGACAACTCCTATCTT ITTTTG \\
\hline FCER1G & ССGGCGGAAGTGAGATCCAGTATAACTCGAGTTATACTGGATCTCACTTCСGTTTTTG \\
\hline BST2 & СCGGCCCAGGAAGCTGGCACATCTTCTCGAGAAGATGTGCCAGCTTCCTGGGTTTTTC \\
\hline A20 esiRNA & Human TNFAIP3 esiRNA (esiRNA1, EHU019961) from MilliporeSigma \\
\hline
\end{tabular}

not been clear which cell surface molecule(s) accounted for the MM inhibitory effect on pDCs. $\mathrm{CDH} 1$ belongs to the cadherin superfamily, and homophilic and heterophilic interaction between these family members mediates cell-cell adhesion (23). It was reported that CDH1 expression was relatively higher in spinal plasma cell myeloma than in simple bone cyst tissues (35), but the significance of this high expression was unknown. $\mathrm{CDH} 1$ is also expressed on DCs and mediates DC-DC interaction (36). Disruption of CDH1-mediated adhesion induces a functionally distinct pathway of DC maturation in order to maintain tolerance rather than to initiate immunity (36). The role of $\mathrm{CDH} 1 \mathrm{in}$ pDCs has not been studied. Here we report what we believe is a novel inhibitory function of $\mathrm{CDH} 1 \mathrm{in}$ modulating $\mathrm{pDC}$ function by attenuating the TLR9 pathway. MM cells utilize the $\mathrm{CDH} 1$ pathway to educate pDCs to promote myeloma pathogenesis and to escape immune surveillance. The universal expression of $\mathrm{CDH} 1$ on different cell types raises the question of how MM cells specifically interact with pDCs. Here, we found that MM cells highly express BST2, and BST2-ILT7 interaction aids in forming MM cell-pDC clusters.

As a classic adherent junction protein, $\mathrm{CDH} 1$ forms a dynamic complex with $\beta$-catenin via its cytoplasmic tail to regulate several signaling pathways, including the Wnt/ $\beta$-catenin, PI3K/Akt, and $\mathrm{NF}-\mathrm{kB}$ pathways (37). A20 is a ubiquitin-editing enzyme, and its activities include deubiquitylase, E3 ubiquitin ligase, and ubiquitin-binding activities. It suppresses TLR pathways by negatively regulating NF- $\mathrm{KB}$ signaling $(38,39)$. We showed that $\mathrm{CDH} 1$ signaling attenuates TLR9 signaling in 2 ways. First, A20 expression was upregulated by activation of $\mathrm{CDH1}$ signaling. A2O negatively regulates the TLR9-mediated NF- $\mathrm{kB}$ pathway by directly inhibiting the E3 ligase activities of TRAF6, TRAF2, and cIAP1 $(38,39)$, and when elevated, A20 quickly inhibits IFN- $\alpha$ production from downstream of the TLR9 pathway in pDCs. Second, we discovered what we believe is a novel function for A20. A20 interacts with TLR9 to ubiquitinate and degrade TLR9, and attenuates the TLR9 pathway by desensitizing $\mathrm{CpG}$ stimulation, which is a very efficient way to terminate TLR9 signaling. $\beta$-catenin may play an important role in A20 activation because its presence was necessary for the interaction of
TLR9 and A20, which emphasizes the importance of activation of $\mathrm{CDH} 1$ signaling for $\mathrm{pDC}$ s to acquire a tumor-promoting phenotype and function.

In summary, we found that the physiological function of pDCs may involve suppressing MM growth. However, MM cells are able to convert pDCs to acquire a tumor-promoting phenotype and function via direct cell contact. CDH1 homophilic interaction induces the degradation of TLR9 and attenuates TLR9 downstream signaling by activating A20. These findings reveal a new mechanism underlying how MM cells escape immune surveillance and provide what we believe are novel molecular targets for improving the treatment of patients with MM.

\section{Methods}

Human samples and cells. Bone marrow aspirates from patients with $\mathrm{MM}$ and healthy donors were used to isolate CD138 ${ }^{+}$ cells with the EasySep Human CD138 Positive Selection Kit II (STEMCELL Technologies, catalog 17877). Human MM cell lines ARP-1, MM.1S, RPMI-8226, and U266, and pDC cell line Gen2. 2 cells that were cultured with MS5 feeder cells, were maintained in RPMI-1640 medium with $10 \%$ fetal bovine serum (Lonza), $100 \mathrm{U} / \mathrm{ml}$ penicillin, and $100 \mu \mathrm{g} / \mathrm{ml}$ streptomycin at $37^{\circ} \mathrm{C}$ and $5 \% \mathrm{CO}_{2}$. Joël Plumas and Laurence Chaperot (Department of Research and Development, Etablissement Français du Sang Rhône-Alpes, Grenoble, France) provided the Gen2.2 cell line. Vk* MYC cells were provided by Leif P. Bergsagel (Mayo Clinic in Arizona, Phoenix, Arizona, USA) and maintained in Rag2 ${ }^{-/-} \mathrm{Il}_{2} \mathrm{rg}^{-/}$mice. Splenocytes containing greater than $50 \%$ of malignant plasma cells were frozen and used for experiments. All other cell lines were obtained from ATCC.

Mice and $V k^{*} M Y C$ MM model. BDCA2-DTR mice (C57BL/6-Tg (CLEC4C-HBEGF) 956Cln/J) were purchased from the Jackson Laboratory and maintained at the Cleveland Clinic animal facility. Mice (male, 6-10 weeks) were injected intraperitoneally with DT (100 ng/ mice) 1 day before $\mathrm{Vk}^{*} \mathrm{MYC}$ cells $\left(1.5 \times 10^{6} /\right.$ mouse $)$ were injected intravenously, and then every other day for a total of 5 times. Mouse serum was collected weekly for protein electrophoresis to monitor the presence of the M-band with the QuickGel SPE Kit (Helena Laboratories, catalog 3405). Briefly, mouse serum samples were diluted 5-fold and a 1- $\mu$ l diluted sample was loaded on the QuickGel SPE Gel (Helena Laboratories, catalog 3505T). Electrophoresis was run at $400 \mathrm{~V}$ for $5 \mathrm{~min}$ utes. Gel was dried with a QuickGel Chamber (Helena Laboratories, catalog 1284) for 15 minutes and stained with Acid Blue for 2 minutes and de-stained the gel with Destain solution. The animal study was approved by the Institutional Review Board of the Cleveland Clinic.

Isolation of pDCs. pDCs from human PBMCs were enriched using the EasySep Human Plasmacytoid DC Enrichment Kit (STEMCELL Technologies, catalog 19062), followed by sorting the $\mathrm{CD} 4^{+} \mathrm{CD} 123^{+}$ $\mathrm{CD}^{-} \mathrm{C}^{-}$population (CD4 and APC-Cy7, BD Biosciences, catalog 557871; CD123 and APC, Biolegend, 306012; CD11C and PE, BD Biosciences, catalog 555392) with the "dumping" channel (all are FITClabeled and from BD Biosciences; $\mathrm{CD}^{+}$, catalog 555339; CD14 ${ }^{+}$, catalog 347493; CD19 ${ }^{+}$, catalog 555412; CD56 ${ }^{+}$, catalog 557699) on a BD Biosciences LSR Fortessa sorter. The purity of pDCs was greater than $95 \%$.

Western blotting. As previously described (40), cells were harvested and washed with PBS twice, lysed in $1 \times$ lysis buffer (Cell Signaling) with protease cocktail (Roche) for 30 minutes on ice, and then centrifuged 
at $12,000 \mathrm{~g}$ for 15 minutes. Supernatant was collected and analyzed by Western blotting with the appropriate antibodies (all from Cell Signaling; TLR9, catalog 13674; TLR7, catalog 5632; MyD88, catalog 4283; $\beta$-actin, catalog 3700; IRF7, catalog 4920; GAPDH, catalog 5174; A20, catalog 5630; ubiquitin, catalog 3936; $\beta$-catenin, catalog 8480).

Viral transduction. shRNAs were synthesized by MilliporeSigma and subcloned into the pLKO.1-GFP lentiviral vector. Viruses were packaged in 293T cells transfected with Lipofectamine 2000 (Thermo Fisher Scientific). Virus-containing culture medium was harvested from day 1 through day 3 , filtered through a $0.45-\mu \mathrm{m}$ filter, and stored at $-80^{\circ} \mathrm{C}$ until used. Cells were mixed with virus and $10 \mu \mathrm{g} / \mathrm{ml}$ protamine sulfate (MilliporeSigma) in a 24 -well plate, then centrifuged for 120 minutes at $500 g$ at $32^{\circ} \mathrm{C}$. After 2 hours of incubation at $37^{\circ} \mathrm{C}$, fresh medium was replaced, and cells were cultured for further assay.

Flow cytometry. For intracellular TLR7 (PE, BD Biosciences, cata$\log 565557$ ) and TLR9 (PE, BD Biosciences, catalog 560425) staining, primary pDCs or Gen2.2 cells were stained for surface marker first and then fixed and stained with the Transcription Factor Staining Buffer Set from Thermo Fisher Scientific (catalog A25866A). For IFN- $\alpha$ staining, mouse splenocytes were stimulated with $1 \mu \mathrm{M} \mathrm{CpG}$ for 1 hour and then blocked by BFA for another 3 hours. Cells were first stained for surface markers (all from Biolegend; CD11C, FITC, catalog 117305; B220, Brilliant Violet 421, catalog 103239; BST2, APC, 127015; SiglecH, Pacific Blue, catalog 129609) and then fixed and stained for IFN- $\alpha$ (Abcam, catalog ab7373) and followed by goat anti-rat IgG secondary antibody (PE, Biolegend, catalog 405406) staining with the BD Cytofix/Cytoperm Kit (catalog 554714). Samples were analyzed by BD Fortessa or Miltenyi MACSQuant systems.

Real-time quantitative PCR. As previously described (40), total RNA was extracted with Trizol, and CDNA was synthesized with M-MLV reverse transcriptase (Thermo Fisher Scientific) and analyzed by real-time quantitative PCR by SYBR Green (Applied Biosystems). The abundance of the mRNAs was normalized to that of the housekeeping gene ACTB. Primer information is provided in Table 1.

Microarray. Cells were cultured under various conditions and then harvested for RNA extraction with Trizol. The microarray was performed with the HumanHT-12 V4.0 Expression BeadChip Kit (Illumina) at Baylor Institute for Immunology Research. The culture conditions were as follows: culture 1: pDCs, 0 hours; culture 2: pDCs and ARP1 mixture, 0 hours; culture 3: ARP-1, O hours; culture 4: pDCs with CpG, 12 hours; culture 5:pDC + ARP1, direct coculture with CpG, 12 hours; culture 6: ARP1 with CpG, 12 hours; culture 7: pDC (CpG), 12 hours, and ARP1 (CpG), 12 hours (mixture); culture 8: [pDCs (in transwell) + ARP1 (well)] coculture with CpG, 12 hours; culture 9: pDCs from culture 8 transwell coculture; culture 10: ARP1 from transwell coculture. Microarray data accession number is GSE113647.

ELISA. pDCs $\left(1 \times 10^{4}\right.$ to $\left.2 \times 10^{4}\right)$ and MM cells were cultured either alone or together at a 1:1.5 (pDC/MM) ratio in half-area 96-well plates under various conditions, or Gen2.2 cells $\left(1 \times 10^{5}\right)$ and MM cells $(1.5 \times$ $\left.10^{5}\right)$ were cultured in 96-well plates. Supernatants were collected and analyzed using human ELISA kits (all from R\&D Systems) according to the manufacturer's protocol.

Mass spectrometry analysis. Gen2.2 cells were stimulated with CpG with IgG control or CDH1 agonist antibody (Thermo Fisher Scientific), and then harvested and lysed for protein electrophoresis. The gel was fixed and sent to the Lerner Research Institute's Proteomics and Metabolomics Core for mass spectrometry.

A2O siRNA transfection. pDCs $\left(5 \times 10^{5}\right)$ were transfected with $1 \mu \mathrm{g}$ human A20 esiRNA (esiRNA1, EHU019961, MilliporeSigma) using the DOTAP Liposomal Transfection Reagent (MilliporeSigma) following the manufacturer's instructions. Knockdown efficiency was detected and confirmed 24 hours after transfection, and cells were used for further experiments.

Statistics. A 2-tailed Student's $t$ test was used for statistical analyses. When more than 2 groups were included in an analysis, Bonferroni's corrected significance level was used. $P$ less than 0.05 was considered to be statistically significant. Results are mean \pm SD.

Study approval. All procedures were approved by the IRB at the Cleveland Clinic.

\section{Author contributions}

QY, YJL, and WF initiated the study. QY and EB designed the experiments and wrote the paper. EB and RL performed most of the experiments and statistical analyses. LCB and HL prepared samples for microarray. PS helped with animal experiments. LCB, $\mathrm{XM}, \mathrm{CH}, \mathrm{QW}, \mathrm{LL}, \mathrm{ZL}$, and JQ provided important suggestions. MY assisted in generating mice.

\section{Acknowledgments}

We thank Joël Plumas and Laurence Chaperot for providing the Gen2.2 cell line and Leif P. Bergsagel for providing $\mathrm{Vk}^{*} \mathrm{MYC}$ cells. We thank C. Talerico, a salaried employee of the Cleveland Clinic, for substantive editing and comments, and the Baylor Institute for Immunology Research for the microarray and data generation. This work was supported by grants from the National Cancer Institute (R01 CA163881, R01 CA200539, R01 CA211073, and R01 CA214811), the Leukemia and Lymphoma Society (6469-15), and the Multiple Myeloma Research Foundation.

Address correspondence to: Qing Yi, 6550 Fannin Street, SM8033, Houston, Texas 77030, USA. Phone: 346.238.5064; Email: qyi@ houstonmethodist.org. Or to: Yong-Jun Liu, 640 Memorial Drive, Cambridge, Massachusetts 02139, USA. Phone: 617.665.4986; Email: Yong-Jun.Liu@sanofi.com. Or to: Weijun Fu, 415 Fengyang Road, Huangpu District, Shanghai, China, 200003. Phone: 86.2181885421; Email: fuweijun@smmu.edu.cn.

QY's present address is: 6550 Fannin Street, SM8033, Houston, Texas 77030, USA. Phone: 346.238.5064; Email: qyi@ houstonmethodist.org.
1. Laubach J, Richardson P, Anderson K. Multiple myeloma. Annu Rev Med. 2011;62:249-264.

2. MacLennan IC. Germinal centers. Annu Rev Immunol. 1994;12:117-139.

3. Kuehl WM, Bergsagel PL. Molecular pathogenesis of multiple myeloma and its premalignant precursor. J Clin Invest. 2012;122(10):3456-3463.

4. Beatty GL, Gladney WL. Immune escape mechanisms as a guide for cancer immunotherapy. Clin Cancer Res. 2015;21(4):687-692.

5. Rabinovich GA, Gabrilovich D, Sotomayor EM. Immunosuppressive strategies that are mediated by tumor cells. Annu Rev Immunol. 2007;25:267-296.

6. Chesi M, et al. AID-dependent activation of a MYC transgene induces multiple myeloma in a conditional mouse model of post-germinal center malignancies. Cancer Cell. 2008;13(2):167-180.

7. Anderson KC. Targeted therapy of multiple myelo- 
ma based upon tumor-microenvironmental interactions. Exp Hematol. 2007;35(4 Suppl 1):155-162.

8. Chauhan D, et al. Functional interaction of plasmacytoid dendritic cells with multiple myeloma cells: a therapeutic target. Cancer Cell. 2009;16(4):309-323.

9. Zheng Y, et al. Macrophages are an abundant component of myeloma microenvironment and protect myeloma cells from chemotherapy drug-induced apoptosis. Blood. 2009;114(17):3625-3628.

10. Görgün GT, et al. Tumor-promoting immune-suppressive myeloid-derived suppressor cells in the multiple myeloma microenvironment in humans. Blood. 2013;121(15):2975-2987.

11. Ramachandran IR, et al. Myeloid-derived suppressor cells regulate growth of multiple myeloma by inhibiting $\mathrm{T}$ cells in bone marrow. JImmunol. 2013;190(7):3815-3823.

12. Li H, et al. Human osteoclasts are inducible immunosuppressive cells in response to $\mathrm{T}$ cell-derived IFN- $\gamma$ and $\mathrm{CD} 40$ ligand in vitro. J Bone Miner Res. 2014;29(12):2666-2675.

13. Feng $Y$, et al. Bone marrow stromal cells from myeloma patients support the growth of myeloma stem cells. Stem Cells Dev. 2010;19(9):1289-1296.

14. Gilliet M, Cao W, Liu YJ. Plasmacytoid dendritic cells: sensing nucleic acids in viral infection and autoimmune diseases. Nat Rev Immunol. 2008;8(8):594-606.

15. Colonna M, Trinchieri G, Liu YJ. Plasmacytoid dendritic cells in immunity. Nat Immunol. 2004;5(12):1219-1226.

16. Hornung V, et al. Quantitative expression of tolllike receptor 1-10 mRNA in cellular subsets of human peripheral blood mononuclear cells and sensitivity to $\mathrm{CpG}$ oligodeoxynucleotides. JImmunol. 2002;168(9):4531-4537.

17. Jarrossay D, Napolitani G, Colonna M, Sallusto F, Lanzavecchia A. Specialization and complementarity in microbial molecule recognition by human myeloid and plasmacytoid dendritic cells. Eur JImmunol. 2001;31(11):3388-3393.
18. Chesi M, et al. Drug response in a genetically engineered mouse model of multiple myeloma is predictive of clinical efficacy. Blood. 2012;120(2):376-385.

19. Jeske S, Pries R, Wollenberg B. CpG-Induced IFN- $\alpha$ production of plasmacytoid dendritic cells: time and dosage dependence and the effect of structural modifications to the $\mathrm{CpG}$ backbone. Nucleic Acid Ther. 2013;23(2):118-124.

20. Chaperot $L$, et al. Virus or TLR agonists induce TRAIL-mediated cytotoxic activity of plasmacytoid dendritic cells. JImmunol. 2006;176(1):248-255.

21. Honda K, Yanai H, Takaoka A, Taniguchi T. Regulation of the type I IFN induction: a current view. Int Immunol. 2005;17(11):1367-1378.

22. Cao W, et al. Plasmacytoid dendritic cell-specific receptor ILT7-Fc epsilonRI gamma inhibits Tolllike receptor-induced interferon production. JExp Med. 2006;203(6):1399-1405.

23. Nagafuchi A, Takeichi M. Cell binding function of E-cadherin is regulated by the cytoplasmic domain. EMBO J. 1988;7(12):3679-3684.

24. Wertz IE, et al. De-ubiquitination and ubiquitin ligase domains of A2O downregulate NF-kappaB signalling. Nature. 2004;430(7000):694-699.

25. Ivashkiv LB, Donlin LT. Regulation of type I interferon responses. Nat Rev Immunol. 2014;14(1):36-49.

26. Swann JB, et al. Type I IFN contributes to NK cell homeostasis, activation, and antitumor function. JImmunol. 2007;178(12):7540-7549.

27. Dunn GP, et al. A critical function for type I interferons in cancer immunoediting. Nat Immunol. 2005;6(7):722-729.

28. Hiroishi K, Tüting T, Lotze MT. IFN-alphaexpressing tumor cells enhance generation and promote survival of tumor-specific CTLs. JImmunol. 2000;164(2):567-572.

29. Mundy-Bosse BL, et al. Myeloid-derived suppressor cell inhibition of the IFN response in tumor-bearing mice. Cancer Res. 2011;71(15):5101-5110.

30. Hashimoto H, Ueda R, Narumi K, Heike Y, Yoshi- da T, Aoki K. Type I IFN gene delivery suppresses regulatory T cells within tumors. Cancer Gene Ther. 2014;21(12):532-541.

31. Sisirak V, et al. Impaired IFN- $\alpha$ production by plasmacytoid dendritic cells favors regulatory T-cell expansion that may contribute to breast cancer progression. Cancer Res. 2012;72(20):5188-5197.

32. Hartmann E, et al. Identification and functional analysis of tumor-infiltrating plasmacytoid dendritic cells in head and neck cancer. Cancer Res. 2003;63(19):6478-6487.

33. Cao W, et al. Regulation of TLR7/9 responses in plasmacytoid dendritic cells by BST2 and ILT7 receptor interaction. J Exp Med. 2009;206(7):1603-1614.

34. Cao W, et al. BDCA2/Fc epsilon RI gamma complex signals through a novel BCR-like pathway in human plasmacytoid dendritic cells. PLOS Biol. 2007;5(10):e248.

35. Wang L, Liu H, He J, Li Y, Xu Y, Chen B. E-cadherin expression and its clinical significance in 41 cases of spinal plasma cell myeloma. Journal of Medical Colleges of PLA. 2009;24(1):56-62.

36. Jiang A, et al. Disruption of E-cadherin-mediated adhesion induces a functionally distinct pathway of dendritic cell maturation. Immunity. 2007;27(4):610-624.

37. Pećina-Slaus N. Tumor suppressor gene E-cadherin and its role in normal and malignant cells. Cancer Cell Int. 2003;3(1):17.

38. Shembade N, Ma A, Harhaj EW. Inhibition of NF-kappaB signaling by A20 through disruption of ubiquitin enzyme complexes. Science. 2010;327(5969):1135-1139.

39. Shembade N, Harhaj E. A2O inhibition of $\mathrm{NF} \kappa \mathrm{B}$ and inflammation: targeting E2:E3 ubiquitin enzyme complexes. Cell Cycle. 2010;9(13):2481-2482.

40. Bi E, et al. Foxo1 and Foxp1 play opposing roles in regulating the differentiation and antitumor activity of TH9 cells programmed by IL-7. Sci Signal. 2017;10(500):eaak9741. 